Chirurg 2013 $\cdot 84: 999$

DOI 10.1007/s00104-013-2633-6

Online publiziert: 11. Oktober 2013

๑) Springer-Verlag Berlin Heidelberg 2013

O. Strobel · M.W. Büchler

Klinik für Allgemein-, Viszeral- und Transplantationschirurgie, Universität Heidelberg

\title{
Die frühe laparoskopische Cholezystektomie ist die Therapie der Wahl bei akuter Cholezystitis
}

anderem ein American Society of Anesthesiologists (ASA) Score $\geq 4$, ein septischer Schock sowie ein Verdacht auf Gallenblasenperforation oder Abszess. In jedem Zentrum waren sowohl die Chirurgen als auch die Gastroenterologen an der Studie beteiligt. In beiden Gruppen erhielten die Patienten das Antibiotikum Moxifloxacin für mindestens $48 \mathrm{~h}$ intravenös. Primärer Endpunkt der Studie war die Morbidität (anhand zuvor festgelegter Komplikationen) innerhalb von 75 Tagen nach Aufnahme des Patienten. Als sekundäre Endpunkte wurden unter anderem Mortalität, Konversionsrate, Liegedauer und Krankenhauskosten untersucht.

\section{Ergebnisse}

Als zentrales Ergebnis war die Morbidität in der ILC-Gruppe signifikant niedriger als in der DLC-Gruppe (11,8\% vs. $34,4 \%$ ). Dabei war in beiden Gruppen eine erhöhte Komorbidität (ASA-Score >2) signifikant mit einer erhöhten Komplikationsrate assoziiert. Mortalität ( $0,3 \%$ in beiden Gruppen) und Konversionsrate (ILC: 9,9\%; DLC: 11,9\%) waren vergleichbar. Die Gesamtliegedauer war bei ILC mit 5,4 Tagen signifikant niedriger als bei DLC mit 10,0 Tagen; bei vergleichbarer postoperativer Liegedauer (ILC: 4,7 Tage; DLC: 4,9 Tage). Dies führte zu signifikant niedrigeren medianen Krankenhauskosten bei ILC (2919 EUR) im Vergleich zur DLC (4262 EUR).

\section{Diskussion}

Die Studie zeigt eindeutig, dass die Morbidität bei früher Cholezystektomie wesentlich niedriger ist als bei primär konserva- tiver Therapie und Cholezystektomie im Verlauf. Zudem hat die frühe Cholezystektomie deutliche ökonomische Vorteile.

$\mathrm{Zu}$ beachten ist, dass in der Studie Patienten mit schwerer Komorbidität ausgeschlossen wurden und die Ergebnisse daher nicht auf solche Patienten übertragbar sind. Ob Patienten mit schwerer Komorbidität von einer präoperativen Optimierung ihres Zustandes durch eine primär konservative Therapie profitieren, muss in weiteren Studien geklärt werden.

Für operable Patienten mit akuter Cholezystitis stellt die frühe Cholezystektomie innerhalb von $24 \mathrm{~h}$ nach Aufnahme die Therapie der Wahl dar.

\section{Korrespondenzadresse}

\section{PD Dr. O. Strobel}

Klinik für Allgemein-, Viszeralund Transplantationschirurgie, Universität Heidelberg, Im Neuenheimer Feld 110, 69120 Heidelberg Oliver.Strobel@med.uni-heidelberg.de

\section{Einhaltung ethischer Richtlinien}

Interessenkonflikt. O. Strobel und M.W. Büchler geben an, dass kein Interessenkonflikt besteht.

Dieser Beitrag beinhaltet keine Studien an Menschen oder Tieren. 